Aus dem Patholog.-Anatomischen Kabinett des Prof. Moiseeff in der Militär-Medizinischen Akademie in Petersburg.

\title{
Zur Frage über die Entwicklung der grossen Gefässe (der Aorta und der Art. brachialis) beim mensch= lichen Embryo.
}

Von

Dr. M. S. Masloff.

Hierzu Tafel XVII.

Ich schicke meiner Darstellung keinen Literaturbericht voraus, da solche in den Arbeiten Aschoffs, Thomas, in den Dissertationen Dobrowolskys, Westphalens u. a. zur Genüge gegeben sind. Das Ziel meiner Arbeit ist die Untersuchung neuen Materials, um ein vollständiges Urteil über die Entwicklung der grossen Gefüsse zu erlangen.

Als Material dienten mir die Kinderleichen der MilitürMedizinischen Akademie und auch die frischen Aborte aus dem Obuchoffschen Krankenhaus zu St. Petersburg. Im ganzen hatte ich zu meiner Verfügung 44 Fälle:

1. 15 jühriges Mädchen, $158 \mathrm{~cm}$ Grösse; 2.5 jähriges Mrädchen, $110 \mathrm{~cm}$; 3. 2 jähriger Knabe, $82 \mathrm{~cm}$; 4. 1jühriger Knabe, $71 \mathrm{~cm} ; 5$. 6 monatlicher Knabe, $60 \mathrm{~cm}$; 6. nengeborener Knabe, $53 \mathrm{~cm}$; 7. 7 monatlicher Abortus, $40 \mathrm{~cm}$; 8.7 monatlicher Abortus, $36 \mathrm{~cm} ; 9$. und 10. zwei Aborten, 6 monatlich, $34 \mathrm{~cm}$ and $28 \mathrm{~cm} ; 11 .-19$. neun Aborten, 5 monatlich, von $27 \mathrm{~cm}, 26 \mathrm{~cm}$; $24 \mathrm{~cm}, 22 \cdot \mathrm{cm}, 21,5 \mathrm{~cm}$, zwei von $20,5 \mathrm{~cm}, 19,5 \mathrm{~cm}, 18, \overline{\mathrm{c}} \mathrm{cm} ; 20 .-33$. 14 Aborten, 4 monatlich, von $17,5 \mathrm{~cm}$, drei Fälle von $17 \mathrm{~cm}, 16 \mathrm{~cm} ;$ zwei Fälle von $15 \mathrm{~cm}$, vier Fälle von $14 \mathrm{~cm}, 13,5 \mathrm{~cm}, 12,5 \mathrm{~cm}$ und $11,5 \mathrm{~cm}$; 34.-38. fünf Aborten, 3 monatlich, von $9,5 \mathrm{~cm}, 9,25 \mathrm{~cm}$, zwei Fälle von $8,5 \mathrm{~cm}$ und $7,5 \mathrm{~cm} ; 39 .-44$. sechs Aborten, 2 monatlich, $6,5 \mathrm{~cm}, 5,8 \mathrm{~cm}$, $5,5 \mathrm{~cm}, 5 \mathrm{~cm}, 3,1 \mathrm{~cm}$ und $1 \mathrm{~cm}$.

Zur Untersuchung wurden kleine Stückchen der Aorta und der Art. brachialis genommen. Bei sehr kleinen Embryonen wurden Schnitte durch den ganzen Körperteil gemacht. Die Stückchen wurden in Formalin und Spiritus fixiert und in Celloidin eingelegt. Färbung mit Hämatoxylin-Eosin, Van Gieson, Hart und Fränkel. Als Ausgangspunkt dienten mir die Aorta eines 15̆jährigen Mädchens und eines 2 jährigen Knaben. Von da ging ich zu den früheren Stadien über. 
Den allgemein angenommenen Daten gemäss unterscheide ich: 1. die Intima, die aus der Schicht der Endothelialzellen, aus der Subendothelialschicht und der Membrana elastica interna besteht; 2. die Tunica media mit der subintimalen Schicht und 3. die Adventitia.

Beim $6 \mathrm{monatlichen} \mathrm{Kinde} \mathrm{hat} \mathrm{die} \mathrm{Aorta} \mathrm{ascendens}$ alle Schichten. In der Subendothelialschicht der Intima ist hier und da eine Anhäufung von Zellen auffallend, deren Kerne runde oder ovale Form haben. Das Protoplasma der Zellen, die nach innen liegen, wird nach Van Gieson gelb gefarbt, die übrige Masse des Protoplasmas trägt den Charakter der Bindegewebszellen. Der Bau der Media und der Adventitia ist ganz gleich dem Verhalten bei Erwachsenen. Jie Subendothelialschicht ist in der Aorta abdominalis nicht gleichmässig: bald ist sie ziemlich breit, bald schmal. Sie besteht aus Bindegewebe, enthält aber auch Muskelfasern in geringer Zahl; ausserdem kommen noch sternförmige Zellen und sehr feine, kurze, elastische Fasern vor. Eine deutliche Membrana elastica interna teilt die Intima von der Media ab. Die elastischen Fasern der Media, die in den Zwischenräumen Muskelzellen und Bindegewebe enthalten, nehmen bei der Adventitia ihr Ende. Die Zahl der elastischen Fasern in der Adventitia ist wenig bedeutend.

Die $\mathrm{A}$ or ta eines $\mathrm{Neugeborenen} \mathrm{zeigt} \mathrm{nichts} \mathrm{Neues.} \mathrm{Bei} \mathrm{e} \mathrm{in} \mathrm{er}$ 9) monatlichen Abortfrucht $(45 \mathrm{~cm})$ war die Intima der Aorta lockerer gebaut, die Subendothelialschicht noch schmäler; sie enthielt feine elastische Fasern: die Membrana elastica interna war nur teilweise ausgesprochen, teilweise zerfiel sie in ein Netz von kleinen Fasern. Näher zur Adventitia wurden die elastischen Fasern der Media immer grösser und dicker; zwischen den Fasern fanden sich ausser den Muskelkernen noch dünne Schichten von Bindegewebe. In der Adventitia waren die elastischen Fasern nicht in grosser Zahl vorhanden. Im unteren Teil der Aorta (Aorta abdom.) enthielt die Subendothelialschicht kleine Netzchen von elastischen Fasern und auch noch Bindegewebe; Muskelzellen und sternförmige Zellen gab es hier nicht. Beim $8 \mathrm{monatlichen} \mathrm{Embryo} \mathrm{ist} \mathrm{die} \mathrm{Subendothelialschicht} \mathrm{noch}$ schwächer, ein schmaler Reif aus Bindegewebe, eine geringe Zahl von feinen elastischen Fasern enthaltend. Die Membrana elastica interna ist in dieser Periode nicht stark ausgesprochen. Im allgemeinen werden die Schichten schmäler, und die Fasern, aus denen sie gebildet sind, werden immer feiner. In den noch früheren Stadien verschwinden einige Elemente ganz. Beim Embryo von $40 \mathrm{~cm} \mathrm{Länge} \mathrm{besteht} \mathrm{die} \mathrm{Intima} \mathrm{nur} \mathrm{aus} \mathrm{der}$ Schicht der Endothelialzellen und aus der Membrana elastica interna, die nicht überall deutlich ausgedrückt ist und in einzelne Fasern zerfällt; auf 
einigen Strecken gibt es eine Subendothelialschicht, bestehend aus Bindegewebe mit elastischen Fasern und Muskelzellen. Die Media und die Adventitia sind noch feiner gebaut. In der Gefässwand der Vasa vasorum kommen noch elastische Fasern vor. Bei der Aorta abdominalis ist die Subendothelialschicht noch schwächer und besteht nur aus Bindegewebe ohne elastische Fasern. Die elastischen Fasern der Media werden feiner und sind ganz locker gelagert. Die Zwischenräume (zwischen den Fasern) sind mit Muskelzellen ausgefüllt, das Bindegewebe ist gering entwickelt.

Ein Embryo von $27 \mathrm{~cm}$ Länge zeigt folgendes. Die Intima der Aorta ascendens besteht aus Hachen Endothelialzellen, die dicht aneinander liegen und runde Kerne haben, ferner aus einer ganz schmalen Subendothelialschicht (Muskel- und Bindegewebe enthaltend) und aus der nicht scharf ausgeprägten Membrana elastica interna. Im unteren Abschnitt der Aorta sind die Zellen des Endotheliums lockerer gelagert: sie stellen platte Zellen dar, die sich durch Fortsätze verejnigen und sich in die Media einschieben. Die Schicht des Bindegewebes unter dem Endothelium ist hier dünner und enthält einige kleine sternförmige Zellen; elastische Fasern gibt es hier keine. Die Membrana elastica interna ist deutlich. Die elastischen Fasern der Media schlangeln sich stark, zwischen ibnen befinden sich ovale oder stubchentörmige Muskelkerne und zarte Schichten von Bindegewebe. In der Adventitia kommen Muskelfasern, elastische Fasern und Vasa vasorum vor.

Ich gehe gleich zur Analyse des Baues der Aorta eines Embryo von $19,5 \mathrm{~cm} L a ̈ n g e$ über, und ich werde mich nur bei den Schichten aufhalten, die einiges Besondere haben. Die Subendothelialschicht ist hier noch vorhanden, aber sehr schmal und enthält elastische Fasern und Muskelfasern. Die Membrana elastica interna ist nicht deutlich. Der Charakter der Muskelkerne der Media ist derselbe wie bei den vorhergehenden Stadien. Das Bindegewebe ist nicht bedeutend, die elastischen Fasern anastomosieren miteinander. In der Adventitia sind noch elastische Fasern und Vasa vasorum vorhanden, diese aber ohne elastisches Gewebe. Die Aorta abdominalis unterscheidet sich darin von der Aorta ascendens, dass die Subendothelialschicht fehlt und die Endothelialzellen direkt auf der sehr deutlichen Membrana elastica interna liegen. In der Adventitia gibt es keine Vasa vasorum Die Arteria brachia lis ist zu dieser Periode völlig differenziert. Sie besteht aus der Schicht der Endothelialzellen mit regelmässigen runden Kernen, aus der deutlichen Membrana elastica interna, aus zwei bis drei Schichten ron feinen, kurzen, elastischen Fasern, aus einer wenig ausgesprochenen Membrana elastica externa und der Adventitia. Die Iruskelkerne der Media sind in zwei Reihen regelmässig kreisförmig angeordnet. 
Die Struktur der Aorta eines Embryo von $17 \mathrm{~cm} \mathrm{Länge}$ bietet wieder etwas Eigenartiges dar. Die Intima der Aorta ascendens besteht aus einer Schicht kolbenförmiger Endothelialzellen mit Fortsätzen und runden Kernen und aus einer klaren Membrana elastica interna, welche in jüngeren Stadien in der oberen Abteilung der Aorta nicht existiert. Nur an einem Bezirk des Gefässes ist die Subendothelialschicht vorhanden, die sehr locker ist und regellos liegende Zellelemente enthält, die sich mit ihren Fortsätzen in die Media einsenken. Bei der Färbung nach Fränkel kann man die Anwesenheit feiner elastischer Fasern feststellen, deren Zahl aber gering ist. Die Kerne der Media haben stabchenförmige Gestalt, sind kreisförmig angeordnet, zuweilen sind sie winkelförmig gekrümmt; die elastischen Fasern schlängeln sich stark, das Bindegewebe ist wenig bedeutend. In der Adventitia sind keine elastischen Fasern vorhanden, wohl aber Muskelfasern; die Vasa vasorum befinden sich noch im IEmbryonalzustand. Die Adventitia geht olne scharfe Grenze ins umgebende Gewebe über. Bei der Lorta abdominalis fehlt die Subendothelialschicht ganz. Die Arteria brachialis hat dieselbe Struktur wie im vorhergehenden Stadium. Wir sehen, dass die Subendothelialschicht langsam verschwindet, zuerst verschwindet sie in der Aorta abdominalis, dann in der Aorta ascendens.

Bei einem Embryo von $14 \mathrm{~cm} \mathrm{Länge} \mathrm{ist} \mathrm{diese} \mathrm{Schicht}$ schon nicht mehr vorhanden, und die Intima besteht aus einer Schicht dicht aneinander liegender Endothelialzellen und einer Membrana elastica interna, die an einigen Orten aus zwei bis drei einzelnen elastischen Fasern besteht. In der Media sind die Fasern weniger gekrümmt und sind bedeutend schmüler. In der Adventitia gibt es keine Vasa vasorum und keine elastischen Fasern. Bei der Aorta abdominalis tritt die Membrana elastica interna scharf hervor, die elastischen Fasern der Media schlängeln sich vielfältig und sind schmäler. Bindegewebe findet sich hier nur wenig. Die Schichten der Arteria brachialis sind in diesem Stadium schon differenziert.

Die Intima der Aorta ascendens eines Embryo von $12,5 \mathrm{~cm}$ Länge besteht aus einer Schicht vieleckiger Endothelzellen mit langen Fortsätzen und mit eiförmigen Kernen, welche zentrale Lage haben. Die Membrana elastica interna ist nicht der ganzen Länge nach vorhanden. Die elastischen Fasern sind in der Intima lockerer angeordnet und anastomosieren miteinander. Die Muskelkerne liegen regellos. Es findet sich nur wenig Bindegewebe In der Adventitia bemerkt man die Embryonalentwicklung der Vasa vasorum. 
Embryo von $9,25 \mathrm{~cm} \mathrm{Länge.} \mathrm{Hier} \mathrm{sind} \mathrm{die} \mathrm{Elemente,} \mathrm{we]che} \mathrm{die}$ Gefässwand der Aorta bilden, noch feiner, sie liegen noch lockerer und treten deshalb dentlicher hervor. Die Endothelialschicht stösst unmittelbar an die Membrana elastica interna an, die an einigen Orten aus dünnen einzelnen Fasern besteht. Die Kerne der Media haben unbestimmte Gestalt, sind gekrümmt und sind in unregelmässigen Reihen angeordnet, welche zwischen den Schichten der dünnen, aber noch reichlichen elastischen Fasern liegen. Nach Van Gieson ist die Färbung der Media nicht so typisch. jedenfalls trägt sie den Charakter der Muskelfïrbung. Das Wiedererscheinen der Subendothelialschicht an einigen Stellen in der oberen Abteilung der Aorta ist hier auffallend. Es ist keine zufällige Erscheinung, denn dasselbe wird an den folgenden noch jungen Embryonen bestätigt. In der Adventitia sind einige elastische Fasern vorhanden.

Beim Embryo von $7,5 \mathrm{~cm}$ Linge liegen die faserigen Elemente noch lockerer. Die Endothelialzellen haben lange Fortsätze, ein blassgefürbtes Protoplasma und runde oder eiförmige Kerne. Die Membrana elastica interna spaltet sich an einigen Stellen in einzelne elastische Fasern. In der Media liegen die Kerne unregelmissig besonders in der Intima und etwas regelmässiger kreisförmig in der Adventitia. Die elastischen Faseln sind dünn und gekrümmt. Die Adventitia besteht aus Bindegewebe mit einer geringen Zahl von Zellen, welche runde Kerne tragen. In der Adventitia finden wir an einigen Orten eine Ansammlung von zwei bis drei bis fünf roten Blutkörperchen (Embryonalstadium der Vasa vasorum), die von Zellen umgeben sind, welche von den umgebenden Gewebszellen sich gar nicht unterscheiden. In der Adventitia gibt es keine elastischen Elemente. In der Aorta abdominalis ist an einigen Stellen unter dem Endothelium ein feinfaseriges Gewebe vor handen welches sich ungefähr auf einen Viertelkreis ausdehnt und eiförmige oder runde Kerne enthält. Diese Bindegewebs schicht (nach Van Gies on) ist ziemlich locker, lässt $Z$ wischenräume erkennen und wird nicht stark gefärbt, obgleich das Bindegewebe des umgebenden Gewebes ganz intensiv rosa gefärbt wird. Der Charakter der Media und der Adventitia ist derselbe wie in der Aorta ascendens.

Die Arteria brachialis unterscheidet sich darin vom vorhergehenden Stadium, dass sie weniger elastische Fasern hat. Die Kerne der Media sind in zwei bis drei Reihen angeordnet, sie sind eiförmig oder stark gedehnt. Nach Van Gieson bekommen die Mediazellen leichte rosa Färbung, dieselbe Färbung, welche das umgebende Gewebe hat, obgleich die Mediazellen im vorhergehenden Stadium die Muskelfärbung aufnahmen. Die Arteria brachialis eines Embryo von $6,5 \mathrm{~cm}$ Länge hat all diese charakteristischen 
Zcichen, die aber noch mehr ausgeprägt sind. Das Protoplasma der Mediazellen ist zweifellos rosa gefärbt (nach Van Gieson) und grün nach Fränkel (d. h. es hat den Charakter von Bindegewebe). Iie elastischen Fasern sind sehr fein wnd kurz und umfassen nur einen Teil des Lumens. Die Membrana elastica interna ist nicht scharf ausgeprägt, wir finden schon ein Anzeichen der Bildung einer Membrana elastica interna, die an einigen Orten als eine Reihe von Punkten erscheint. Es ist unmöglich, eine Adventitia zu unterscheiden.

Den nächsten Embryo der Grösse nach (5.8 cm Länge) werde ich ausführlicher beschreiben. In der Aorta ascendens besteht die Intima aus einer Reihe kolbenförmiger Endothelialzellen mit grossen runden oder eiförmigen Kernen; der aussere Teil dieser /ellen erscheint als stark gezogene Linie, wälırend der innere 'Teil unregelmässig ist. Unter dem Endothelium befinden sich an einigen Orten rote und weisse Blutkörperchen, welche kerne enthalten und einzeln oder in kleinen Gruppen liegen; ferner gibt es hier noch ein wenig Fasergewebe. Olne eine deutliche Membrana elastica interna geht die Intima in dic Yedia über. In dieser sind die elastischen Elemente sehr dünr und in grosser Zahl vorhanden, sie sind unregelmässig angeordnet, kreisförmig und bilden Schlingen. Nach aussen sind die elastischen Fasern grösser und liegen regelmässiger; in del Nähe der Adventitia haben sie ihr Ende. Die Mediakerne sind grösstenteils rund oder eiförmig; nach Van Gieson wird die Media in unbestimmt gelber Farbe gefürbt, und man kann kein bestimmtes Ürteil über den Charakter der Mediazellen abgeben. Die Adventitia ist faserig, enthält rote Blutkörperchen, die noch nicht ganz von Zellen umschlossen sind. Sie besteht aus Bindegewebe (die Färbung ist hier aber nicht charakteristisch).

In der Bauchaorta desselben Embryo liegen die Endothelialzellen noch lockerer. Zwischen den Zellen und der klaren Membrana elastica interna kommen rote und weisse Blutkörperchen vor. Die Mediakerne liegen regellos und sind ungeführ in 20 Reihen angeordnet. Die Arteria brachialis erscheint als ein Lumen, das von roten Blutkörperchen ausgefüllt ist und ums Lumen herum sind ringförmig Kerne ausgelegt von eiförmiger oder runder Gestalt in zwei bis drei Reihen, die sich sehr wenig, nur der Lage nach, vom umgebenden Gewebe unterscheiden. Dieses Gewebe trägt den Charakter von Bindegewebe (wird nach Van Gieson rot, nach Fränkel grün gefärbt). Zwischen den ein- 
zelnen Reihen von Zellen kann man die Anfangsbildung der elastischen Fasern (Färbung nach $\mathrm{H}$ art) bemerken, die als punktförmige Bildungen erscheinen (im Querschnitt).

Nun sind wir zum Stadium der unvollstandigen Differenzierung der Arteria brachialis gekommen, die Mediazellen tragen noch den Charakter des Bindegewebes und unterscheiden sich wenig vom umgebenden Gewebe, wo das elastische Gewebe sich erst zu bilden anfüngt. Wir gebeu jetzt zu den früheren Entwicklungsstadien der Aorta über. Bei einem Embryo von $5 \mathrm{~cm}$ Lànge ist der Bau der Gefässwand der Aorta derselbe wie in der vorhergehenden Periode.

Einfacher ist der Bau der Aorta bei einem Embryo v on $3,1 \mathrm{~cm} \mathrm{~L}$ änge. Hier fängt die Differenzierung der Elemerte an, und so möchte ich dies Stadium eingehender schildern.

Das Lumen der Aorta ascendens ist von kernhaltigen roten Bhutkörperchen ausgefüllt. Die Intima besteht aus Zellen, welche dem Scheine nach sich wenig von den Mediazellen unterscheiden; nur ihre Lage zwingt sie für Endothelialzellen zu halten. Die Endothelialkerne sind grösstenteils eiförmig, werden gut gefürbt; das I'rotoplasma ist nicht reichlich, es umgibt den Kern wie ein Reif. Die Umrisse der Zellen sind nicht scharf; einige Zellen, die zum Gefüsslumen gerüclit sind, sind eiförmig, haben Fortsittze. mit welchen sich die Zellen vereinigen und sich in die Media einsenken. Es folgen ohne scharfe Grenze einige Reihen rou Zellen, welche Kerne von verschiedener Crrösse und Form haben und ohne bestimmte Ordnung liegen; der grüsste Teil dieser Zellen liegt kreisförmig; zwischen den einzelnen Zellen bleiben Spalten. Es gelingt nicht, hier die Grenzen gegen die Adventitia zu bestimmen. Es ist auffallend, dass nach aussen hin die Kerne lïnger werden und die Zwischensubstanz schwächer gefürbt wird. Nach Van Gieson nimmt dieses ganze Gewebe unbestimmt grau-gelbe Farbe an; das schwach rosa gefürbte Bindegewebe kommt nur an der Peripherie des Gefatsses in dem umgebenden Gewebe vor. Bei der Fürbung nach Fränkel bekommt man auch unbestimmte Tönung. Schon in diesem Stadium kann man die Anwesenheit elastischer Fasern konstatieren, die sehr fein sind. Sie verlaufen als geschlängelte Fäden zwischen den Zellelementen der künftigen Media. Man kann ihrer bis acht Zellen zählen; sie teilen alle Reihen der Kerne voneinander 
ab. Sie sind noch sehr fein und kurz; einige erreichen die Länge von einem Viertelkreis. Die Fasern, die näher zur Intima. liegen, sind feiner, an einigen Stellen sind nur Reihen von Punkten sichtbar. Dank der Anwesenheit dieser primitiven elastischen Fasern kann man ungefähr die Grenzen der Media bestimmen. Die Adventitia kann man nicht gut rom umgebenden Gewebe trennen. Der Bau der Lungenarterie ist derselbe, nur ist die Zahl der elastischen Fasern kleiner. Um die Bauchorta untersuchen zu können, musste ich Schnitte durch den ganzen Embryo machen.

Dort, wo nach bestimmten topographischen Daten wir die Aorta $z u$ sehen erwarten, sehen wir ein unregelmässiges Lumen, ganz ausgefüllt von kernhaltigen roten Blutkörperchen. Das Lumen ist von einigen Zellenlagen umgeben, die Kerne enthalten. Es gelingt nicht, einzelne Schichten zu unterscheiden, denn die Kerne sind nicht charakteristisch und sind alle einander ïhnlich. Die Blutkörperchen, welche Kerne enthalten (es sind Erythrozyten, denn sie baben gelben Farbenton), haben nuregelmässige, vieleckige oder eiförmige Gestalt. An diese Blutkörperchen stossen Reihen von Zellen an, welche unregelmässig angeordnet sind und vieleckige oder eiförmige Gestalt und auch Fortsatze haben, iln Protoplasma fürbt sich schwach und der Kern ist gross und eiförmig; diese Zellen vereinigen sich miteinander. Einige von ihnen sehen aus, als ob sie sich in die Masse der Blutkörperchen vorschieben, so dass es hier keinen dichten endothelialen Ring gibt. Die nächsten Zellenreihen liegren regelmässiger; sie gehen ohne jegliche Grenze ins umgebende Gewebe über. Nach Van Gieson wird dieses Gewebe unbestimmt gefärbt und auf dem Schnitt durch den ganzen Embryo finden wir nur sehr wenig rosa gefärbtes Bindegewebe. Bei der Färbung nach Hart und Frankel kann man schon zweifellos elastische Fasern konstatieren, die noch im Embryonalzustand sind. Man kann einige Reihen von diesen Fasern unterscheiden. Die Fasern sind sehr fein; grösstenteils sehen wir nur eine Reihe von Punkten, so dass wir einen Eindruck bekommen, als ob die Fäserchen aus dem Zusammenfluss einzelner Punkte, die zwischen den Zellen liegen, entstehen. Die ersten elastischen Fasern und Punkte sehen wir nicht an dem Orte, wo die Membrana elast. int. ist, sondern weit vom Lumen entfernt, durch 2 bis 3 Reihen von 
Kernen getrennt. Die ersten elastischen Fasern sind sehr fein und erst nach aussen hin liegen sie dicht und werden länger. Die Fäserchen folgen den Windungen der Zellen und umgeben das Gefässlumen. An einigen Orten sehen sie aus, als ob sie sich spalten; zur Peripherie hin werden sie wieder kleiner und feiner, und sie haben ihren Anfang wahrscheinlich im Zentrum der künftigen Media. In dem Gewebe, wo die Anordnung der Kerne regelmässig kreisförmig ist, kommen die elastischen Fäserchen nicht mehr vor. Es ist zweifellos, dass auf dem Querschnitt die Fäserchen sich aus dem Zusammentluss einzelner elastischer Körnchen bilden. Im nächsten Stadium, dem eines Embryo von $1 \mathrm{~cm}$ Länge finden wir keine Gefässe, denn auf dem Querschnitt gibt es nur Embryonalgewebe, das Kerne enthält.

Im Jahre 1911 hat Bjorling mitgeteilt, dass er in der Aorta eines Erwachsenen ein Gewebe gefunden habe, das er "mucoides Gewebe" nannte. Bjorling hat die Furbung mit polychromischem Methylenblau (verinderte Methode von Ippa) mit der Differenzierung in Anilinöl, dann mit Alaun gesättigt, angewandt, und er hat gefunden, dass es in der Media hauptsächlich in ihrem inneren Teil, ein dünnes fisseriges, locker liegendes Gewebe gibt, das die rote larbe autnimmt, während das gewöhnliche Bindegewebe blau gefürbt wird. Da dieses Gewebe der Färbung nach dem Schleimgewebe nahestand, so nannte er es mucoides Bindegewebe. So besteht das intramusculare Gewebe aus dem gewölınlichen Bindegewebe und aus dem mukoiden Bindegewebe, das als sehr feine Netzchen auf der homogenen Substanz zwischen den Muskelzellen, besonders in der Nähe der Intima, liegt. Dieses Gewebe fand der Autor in allen grossen und mittelgrossen Gefässen. Bei Arteriosclerosis und Syphilis ist dieses Gewebe velmehrt. Es erschien interessant, die Entwicklung dieses eigentümlichen Gewebes in den Gefässwanden von Embryonen zu untersuchen. Zu diesem Behufe wurde ein Teil von Embryonen in Sublimat fixiert und unter Paraffin gesetzt. Bei dieser Fixierung bekam man eine starke Polychromasie. Parallel der Färbung mit dem polychromischen Methylenblau wurde ein Teil von Schnitten mit 'lhionin und Toluidin gefărbt. Im postfötalen Leben ist das mukoide Gewebe in der Aorta bedeutend. Das mukoide Gewebe liegt in der Media in ziemlich dicken roten Schichten zwischen den elastischen Fasern; die grösste Masse 
befindet sich in der Nähe der Intima; es findet sich anch selbst in der Intima als ein schmaler Streifen nahe dem Lumen. Diese Schichten folgen der Reihe der blauen Schichten, die aus einfachem Bindegewebe gebildet sind. In der Adventitia ist das mukoide Gewebe gering. Bei der Aorta abdominalis ist es etwas weniger entwickelt. Interessant erscheint die Menge dieses $(\dot{e}$ webes bei einem Embryo von $28 \mathrm{~cm}$ Länge. Hier haben die Gefässwände der Aorta an zwei Stellen Verdickungen, und an diesen Stellen ist das mukoide Gewebe stark vergrössert: es liegt als grosses faseriges Netz in der Media zwischen den einzelnen elastischen Fasern. Das einfache Bindegewebe ist hier gering. In den früheren Stadien ist sein Vorkommen dasselbe. Dieses Gewebe ist auch in der Subendothelialschicht der Aorta abdominalis als eine dünne faserige Schicht zu finden. In der Media liegt es hauptsaichlich näher zur Intima, in der Adventitia kommt es nicht vor. Es ist auffallend, dass in den Serien desselben Alters die Masse des mukoiden Gewebes dort wachst, wo es eine Verdickung der Schicht in der Intima oder der Vedia gibt. Beim Embryo von $20 \mathrm{~cm}$ Länge gibt es mukoides (iewebe in der Intima nur teilweise, in der unteren Abteilung der Aortir ist es gar nicht vorhanden.

Beim Embryo von $15 \mathrm{~cm}$ Länge ist dies Gewebe an der Stelle der Verdickung der Subendothelialschicht und auch als eine Subintimalschicht vorhanden; in der Media gibt es nur feine dünne Schichtchen desselben. In der Aorta ascendens eines Embryo von $13 \mathrm{~cm}$ Länge liegt dieses Gewebe als eine dünne Schicht in der Media ganz nahe der Intima, in der Bauchaorta. ist es gering.

Beim Embryo von $9,5 \mathrm{~cm}$ Länge gibt die Färbung nicht mehr eine sichere Reaktion, nur in der Aorta ascendens bekommen einzelne feine Fäserchen unbestimmt rote l'arbe.

Ehe ich zum Überblick der bekommenen Daten übergehe, gebe ich die Dicke der Gefässwände und der einzelnen Schichten in Millimetern an:

Da meine Untersuchungen auf ein ziemlich grosses Material sich erstrecken, und ich verschiedenartige Färbungen anwandte, ist es mir gelungen festzustellen, dass die Differenzierung der einzelnen Schichten und Elemente viel früher eintritt, als es bisher bekannt war. Schon beim Embryo von $3,1 \mathrm{~cm}$ Länge 
Die Entwicklung der grossen Gefässe beim menschlichen Embryo. 361

\begin{tabular}{|c|c|c|c|c|c|c|c|c|c|}
\hline \multirow[b]{2}{*}{$\begin{array}{c}\text { Lănga des } \\
\text { Embryo } \\
\mathrm{cm}\end{array}$} & \multirow[b]{2}{*}{ Alter } & \multicolumn{4}{|c|}{ Aorta ascendens } & \multicolumn{4}{|c|}{ Aorta abdominalis } \\
\hline & & $\begin{array}{c}\text { Die ganze } \\
\text { Gefäss- } \\
\text { wand }\end{array}$ & Intima & Media & Adventitia & $\left|\begin{array}{c}\text { Die ganze } \\
\text { Gefăss- } \\
\text { wand }\end{array}\right|$ & Intima & Media & Adventitia \\
\hline 3,1 & 2 Mon. & & & & & 0,166 & & & \\
\hline 5,8 & 3, & 0,249 & 0,016 & 0,166 & 0.058 & & 0,016 & 0,116 & \\
\hline 7,5 & 3 & 0,266 & & 0,182 & $0,08: 3$ & & & & \\
\hline 9.25 & 3 & 0,308 & & 0,241 & 0,066 & 0,233 & & 0,143 & 0,066 \\
\hline $11, \overline{5}$ & 3 & 0,299 & & 0,216 & 0,092 & 0,2333 & & ), 166 & 0,067 \\
\hline 12,5 & 3 & 0,416 & & 0,333 & 0,092 & 0,249 & & 0,166 & 0,05 \\
\hline 14,0 & 3 & 0,427 & & 0,316 & 0,083 & 0,299 & & 0,183 & 0.066 \\
\hline 17,0 & 3 & 0,416 & & 0,316 & 0,099 & 0,299 & & 0.2333 & 0,066 \\
\hline $19, \overline{5}$ & 3 & 0,$466 ;$ & & 0,383 & 0,099 & 0,299 & & 0,166 & $0,13: 3$ \\
\hline 27,0 & 3 & $0,8: 33$ & & 0,566 & 0,249 & 0,666 & & 0.499 & 0,166 \\
\hline 36,0 & 7 & $\cdot 1,083$ & & 0,751 & 0,333 & 0,649 & & 0,366 & 0,266 \\
\hline 40,0 & 8 & 0,449 & & 0,249 & 0,182 & $0,2 ! 9)$ & & 0,199 & (0,13:3 \\
\hline 45.0 & 9 & 0,783 & & 0,566 & 0,233 & 0,666 & & 0,499 & 0,199 \\
\hline 60,0 & $6 \quad "$ & 0,883 & & 0,783 & 0,101 & 0,749 & & $0,32 \%$ & 0.350 \\
\hline 82,0 & $2 \mathrm{~J} .1 \mathrm{NI}$. & 0,8666 & & 0,649 & 0,166 & 0,8333 & & 0.483 & 0,3333 \\
\hline
\end{tabular}

kann man dem Aussehen nach die Zellen des Endotheliums von dem umgebenden Gewebe unterscheiden, in den späteren Stadien jedoch, von $5 \mathrm{~cm}$ Länge an und mehr, sondern sich die Zellen des Endotheliums rom übrigen Gewebe ab und werden charakteristisch. Es ist auffallend, dass in der Intima schon in den früheren Stadien der Entwicklung Blutkörperchen vorhanden sind. Es ist dies kein Zufall; dafür spricht, dass man die Blutkörperchen in allen früheren Stadien bis zum Embryo von 12,S cm Lünge finden kann.

Es gibt viele Meinungen in bezug aut die Subendothelialschicht. Einige Autoren (Thoma) schreiben die Erscheinung dieser Schicht dem postfötalen Leben zu. Andere (Aschoff) fanden diese Schicht auch früher, aber nicht früher als im 6. Nonat $(28 \mathrm{~cm})$. In bezug auf den Bau dieser Schicht und ibre Bedeutung stimmen ebenso die Meinungen nicht überein. Unsere Beobachtungen haben gezeigt, dass diese Schicht bereits während des fötalen Lebens sich zeigt, anfänglich natürlich schwächer entwickelt.

Es ist erforderlich zu sagen, dass die Gefässentwicklung bei den einzelnen Embryonen unregelmässig verläuft, so dass die Aorten zweier Embryonen, welche dieselbe Grösse haben, ein etwas verschiedenes Bild geben können. Die Subendothelialschicht 
ist hauptsächlich eine Bindegewebsschicht, zu welcher sich in den späteren Stadien elastische Fasern, Muskelfasern und liernbildungen gesellen. Die elastischen Fasern sind dort in grosser Zahl vorhanden, wo die Membrana elastica interna nicht ausgeprägt ist. In den frülieren Perioden ist diese Schicht schmäler, oder sie umgibt nur einen Teil des Lumens. Am allerersten verschwindet sie in der Aorta abdominalis eines Embryo von $17,0 \mathrm{~cm}$ Länge und in der Aorta ascendens verschwindet sie beim Embryo von $14,0 \mathrm{~cm}$ Länge. Es ist interessant, dass in der früheren P'eriode der Entwicklung in dieser Schicht schon die Muskelfaterm dominieren.

Bemerkenswert ist ferner, dass bei den noch jüngeren Embryonen die Subendothelialschicht wieder erscheint (siehe die Aorta abdom. eines Embryo von 7,5 cm Lünge), aber die Möglichkeit ist nicht ausgeschlossen, dass in diesem Fall eine frühere individuelle Entwicklung stattgefunden hat. Jedenfalls spricht dieses Falitum dafür, dass die Subendothelialschicht sehr früh erscheinen kann. schon im 2. Monat. Es ist ferner interessint, dass dort, wo diese Schicht stärker ausgeprïgt ist und eine Verdickung der Getässwand bildet, in ibr das mucoide Gewebe prïlominiert. Jie meisten Autoren finden, dass die Membrana elastica interna zur Intima gehöre. Bis jetzt dachte man, dass ihre elastischen Elemente früher als alle anderen elastischen Fasern der Media erscheinen. Aschoff, der mit den jüngsten Embryonen zu tun hatte (von $5,6 \mathrm{~cm}$ Länge an), erklärt es folgenderweise: schon bei einer Frucht von $5,(i \mathrm{~cm}$ Lange ist vom Endothelium nach aussen hin eine glasähnliche klare zarte Haut vorhanden, ein Anfang der Membrana elastica interna - sie trägt aber noch nicht den Charakter eines elastischen Elementes. Im weiteren verschwindet sie, und erscheint erst im 4. Monat wieder (beim Embryo von 16,7 cm Länge) als eine typische Membran, welche charakteristische Färbungen annimmt. Ferner wird sie nach und nach immer dicker. Nach der Meinung Aschoffs und anderer ist die Membrana elastica interna das erste elastische Gebilde; die anderen elastischen Fasern entwickeln sich später, und ihre Entwicklung geht von innen nach aussen. Diese Meinung Aschoffs, die auch andere angenommen haben, kann durch meine Beobachtungen nicht bestätigt werden. Zunächst muss ich wieder hervorheben, dass die individuelle Entwicklung der Aorta 
bei den einzelnen Embryonen nicht immer eine gleiche ist. Icl hatte Gelegenheit, Beobachtungen über einige Embryonen desselben Alters (del'selben Länge) anzustellen, und ich konnte konstatieren, dass in einigen Fällen die Entwicklung der einzelnen Elemente viel fruher eintreten kann, als in anderen Fillen. Icls konnte die Membrana elastica interna schon bei Embryonen sehr frühen Alters konstatieren; bei einem Embryo von $5,0 \mathrm{~cm}$ Lïnge war die Membrana elastica interna del Bauchaorta zweifellos stark ausgeprägt - in der Aorta ascendens ist die Membrana elastica interna zu dieser Zeit gewöhnlich noch nicht erkennbar - es existieren nur einzelıe feine Fäserchen. In diesem Falle trug die Membrana elastica interna von Anfang an zweifellos den Charakter des elastischen Gewebes.

In den jungen Stadien muss man stärker färben, um die nötige Farbenstufe zu bekommen. Ungeachtet der gr'ossen Zahl von Fällen habe ich nie jene glasähnliche, aber unelastische Membran bemerlit, welche A s ch of $f$ erwihnt. Die Unvollkommenheit des Verfahrens von $\mathrm{M}$ an $\mathrm{chot}$, welches $\mathrm{Asch}$ off angewandt hat, erklït dieses. Zweifelhaft ist es, zu denken, dass diese unelastische Membrana verschwinde, um spaiter wieder, aber jetzt als eine elastische Vembrana, zu erscheinen. Die Sache muss man sich so vorstellen, dass in einigen Fällen. die A s c h off $\mathrm{zu}$ seiner Velfügung lratte, die Entwicklung des elastischen Gewebes später eintrat, und ausserdem konnte das feine elastische Gewebe, welches die Fürbung schwach aufnimmt, bei seiner Färbungsmethode unbemerlit bleiben. Ich stelle mir die Sache so vor: die Membrana elastica interna oder das Netz von Fäserchen, welche der Membrana elastica interna entspricht, entwickelt sich gewöhnlich sehr früh, im 2. Monat (Embryo von $5,0 \mathrm{~cm}$ Lünge). Von Anfang an trägt die Nembrana elastica interna zweifellos einen elastischen Charakter. Im weiteren tritt nach und nach ilhre Verdickung ein. In einigen Fallen kann die Entwicklung der Membran und des ganzen elastischen Gewebes ein wenig später eintreten. Am starksten ist die Membrana elastica interna in der Aorta abdominalis; in der Aorta ascendens fehlt sie gewöhnlich, und dort ist die Grenze der Intima gegen die Media nicht scharf ausgesprochen, da an der Stelle der Membrana elastica interna nur kleine einzelne Fäserchen gelagert sind. Noch in der Periode des fötalen Lebens tritt za- 
weilen die Zerspaltung der Membrana elastica interna in zwei bis drei Fäserchen oder auch in ein Netz von Fäserchen ein (Embryo von $7,5 \mathrm{~cm}$ ); aber das ist keine bestandige Erscheinung und bezieht sich nur auf einen kleinen Teil der Membran.

An meinen Fallen kann ich die Meinung nicht bestatigen, dass die Membrana elastica interna das erste elastische Gewebe ist, und dass die Entwicklung der elastischen Fasern von imnen nach aussen geht. $\mathrm{Zu}$ meiner Verfügung hatte ich die Aorta abdominalis und die Aorta ascendens eines Embryo ron $3.1 \mathrm{~cm}$ Länge. Auf diesen Praparaten, die nach Hart und Friakel gefärbt waren. tritt die Entwicklung der elastischen Fasern deutlich hervor. Wir sehen, dass die ersten vollstandig gebildeten elastischen Fasern weit von der Intima in der Mitte der kiüftigen Media erscheinen; näher zur Intima und zur Adventitia bemerlit man eine Reihe von Punkten an der Stelle der küuftigen Faser oder der Jembran: jedenfalls erscheinen die ersten elastischen Fasern und Punkte nicht an der Stelle der Membrana elastica interna. Die Beobachtung erlaubt uns, anzunehmen, dass dit Entwicklung des elastischen Gewebes von der Peripherie zum Zentrum geht, oder richtiger gesagt, von der Mitte der Schicht. welche der Media entspricht, aus. $/$ u meinem Bedauern muss ich sagen, dass ich keine Längsschnitte machen konnte und deshalb nicht bestimmt sagen kann, ob diese Punkte nur Querschnitte der Länge nach verlaufender Fäserchen oder punktförmige elastische Bildungen sind, welche an der Peripherie der /ellen erscheinen; jedenfalls ist es zweifellos, dass die Fasern und die Membran, welche wir auf den (querschnitten sehen, an dem Zusammentluss einzelner Punkte entstehen. Yon diesem Standpunkt aus erscheint die Meinung Aschoffs über die glasïhnliche Membrana irrig. Unsere Meinung betreffs des Befundes bei dem Embryo von $3,1 \mathrm{~cm}$ Lünge wird auch an den nächst ailteren Embryonen bestätigt, wo es viele elastische Fasern gibt; dort sehen wir, dass die grössten Fasern in der Mitte der Media vorkommen, in der Intima sind sie bedeutend schmaler, kürzer und schlängeln sich mehr.

Je mehr sich der Embryo entwickelt, desto grösser wird die Zahl und die Starke der Fasern, und sie beginnen die zwischen ihnen liegenden Elemente zusammenzupressen. Die Faserr sind in der Intima sehr gekrümmt und können mit- 
einander anastomosieren, indem sie Netze bilden, in deren Spalten Muskelkerne und das Bindegewebe liegen.

Nach Morpurgo und anderen Autoren entwickelt sich die Media von innen nach aussen, denn man beobachtet zuerst die Vermehrung der Kerne nabe bei der Intima. Anfünglich ist sie aus Bindegewebe gebildet. Es gelang bisher Niemandem, die Anfangsbildung der Muskelzellen zu beobachten; bei Yorpu ro fehlen die Zwischenstadien, Asch off beschätigte sich gar nicht mit der Differenzierung der Gewebe. Nach meinen Beobachtungen entwickelt sich die Media aus dem Embryonalgewebe, welches dem Aussehen nach sich vom Bindegewebe gar nicht unterscheidet. Dieses Gewebe nimmt in den frühen Stadien bei der Aorta die Färbung mit Fuchsin (nach Van Gieson) nicht an, erhalt nur eine unbestimmt gelbgraue Tönung. Die Arteria brachialis hat in derselben Periode die Farbe des Bindegewebes, und der Charakter der Kerne ist ganz gleich dem Charakter der Kerne des umgebenden Gewebes; so können wir annehmen, dass die Media der Aorta auch den Charakter des Bindegewebes triigt. Im weiteren haben wir zweifellos Muskelfasern. Ausser dem Muskel- und elastischen Gewebe treten als bestïndiges Eigentum der Media von den allerfrühesten Perioden an $12.5 \mathrm{~cm}$ Länge) dünne Schichten des typischen Bindegewebes und des mukoiden Gewebes hervor, die Menge dieses Gewebes wächst mit dem Alter des Embryo. Die Grenze zwischen der Media und der Adventitia ist nicht scharf und wird durch das Enterbrechen der grossen elastischen Platten bezeichnet; diese Grenze kann man schon beim Embryo von $5,8 \mathrm{~cm}$ Länge erkennen.

Was die Adventitia betrifft, so kann man sie vom ungebenden Gewebe in frühen Perioden gar nicht abgrenzen, und erst yom 3. Monat an beginnt diese Abgrenzung. Die Adventitia trägt bindegewebigen Charakter, obgleich sie in den frühen Stadien die Fuchsinfarbung schwach aufnimmt. Die Vasa vasorum erscheinen sehr früh: den Anfang kann man schon im 4. Monat sehen (12,5 cm Lange). Sie stellen anfangs nur eine Anhäufung von Blutkörperchen dar, im weiteren werden sie von regelmässigen Zellenreihen umgeben, welche die Gefässwände der Vasa vasorum bilden. Im 6. Monat erscheinen in ibren Gefasswänden elastische Fäserchen. Was die elastischen Elemente der Adventitia anbetrifft, so erscheinen sie, Asch offs Meinung entgegen, schon sehr früh 
in der Aorta, im 3.-4. Monat $(9,5 \mathrm{~cm})$, sind aber eine unbeständige Erscheinung, denn bei anderen Embryonen, in spüteren Stadien sogar, sind sie in der Adventitia nicht vorhanden, aber vom 5. Monat an ist ihre Anwesenheit eine bestandige. Was die Muskelfasern der Adventitia betrifft, so ist ihre Anwesenheit auch zweifellos, doch nicht bestündig. Zum erstenmal sahen wir sie beim Embryo von $17 \mathrm{~cm}$ Lünge (4 Monate).

Die Entwicklung der Arteria brachialis ist einfacher. Noch beim Embryo von $5,8 \mathrm{~cm}$ Länge können wir bloss nach dem Aussehen der Zellenelemente nicht von einer Endothelialschicht reden, denn alle Zellen, welche das mit Blutkörperchen gefüllte Lumen umgeben, und auch ihre Kerne sind einandel änlich. Der Interschied ist nur der, dass die ersten drei Reihen regelmissiger kreisförmig liegen. Sie haben ohne Zweifel den Charakter von Bindegewebe (nach Van Gieson). In diesem Stadium schon haben wir Gelegenheit, elastische Fasern zu beobachten, welche auf den Querschnitten als feine schwarze Punkte dargestellt sind (nach $\mathrm{Har}$ ), und die ersten elastischen Elemente erscheinen weit von der Intima entfernt. In den nächsten Perioden tritt die Differenzierung deutlicher hervor: es erscheinen die Membrana elastica interna, die unklare Membrana elastica externa und drei Reihen von feinen elastischen Faseln zwischen den Reihen der Nediaelemente, welche bis zum 4. Monat den Charakter von Bindegewebe behalten. Wieder muss gesagt werden, dass zuweilen die Erscheinung der elastischen Fasern spiiter eintritt (Embryo von $7,5 \mathrm{~cm}$ Lünge). Im weiteren nehmen die Mediazellen die Muskelfürbung an, die Membrana elastica interna und externa treten deutlicher hervor. Die Mediakerne dehnen sich, die Zahl der Schichten wird wach und nach grösser. Dieser Charakter der Media bleibt auch nach der Geburt derselbe. Die Differenzierung der Adventitia in der Arteria brachialis tritt am Ende des 3. Monats ein. In der Adventitia unterscheidet man eine ïussere und eine innere Schicht, in der inneren erscheinen vom 6. Monat an die elastischen Fäserchen.

Herrn Professor Moïseeff sage ich für die Stellung der Aufgabe und für seine beständige Leitung bei deren Bearbeitung verbindlichsten Dank. 
Die Entwicklung der grossen Gefässe beim menschlichen Embryo. 367

\section{Literaturverzeichnis.}

1 Aschoff: Beitrag zar Entwicklungsgeschichte der Arterien beim menschlichen Embryo. Morph. Arb., herausgeg. v. Schwalbe. Jena 1893, Bd. II.

2. Derselbe: Über die Entwicklung, Wachstums- und Altersvorgänge an den Gefïssen von elastischem und muskulïrem Typus. Jena, Fischer. 1908.

3. Bjorling: Über mukoides Bindegewebe. Virchow s Arch. 205, 1911.

4. Bory: De l'éditic. élast. dans les artires de l'embryon. Comp. r. soc. biol. 67 , cmp. 644 .

5. Bonnet: Über den Bau der Arterienwand. Ref. Jahresber. d. Anat. u. Entwickl., N. F., 13. März 1907, S. 240.

b. Dobrowolsky. N.: Über die Verïnderungen der Arterien nach dem Alter. Diss., St. Petersburg 1902.

7. Grunstein: Über den Bau der grösseren menschlichen Arterien in verschiedenen Altersstufen. Arch. $f$ mikr. Anat., Bd. 47, 1896.

8. Morpurgo: Über die Fntwicklung der Arterienwand Sitzungsher. d. Hathem.-Naturw. Kl d. Kaiserl. Akad. d. Wissensch., Bd. XC, 1885 (cit nach Dobrowols ky).

9. Kohle: Wachstum und Altern der grussen Gefaisse und ihre Bezieluungen zur Pathologie des Gefibsystems. Jahresber. d. Anat. 12. Entwicklungsgeschichte. N. F., 13. Mïrz 1907, emp. 213.

10. S $\mathrm{ch} \mathrm{morl}$ : Die pathologisch-auatomischen Untersuchungsmethoden. 1905.

11. Sobole f : Dic Grïde d. pathologisch-histologischen Technik. St. Petersburcr 1910 .

12. Thoma: Über die Abhängigkeit der Bindegewebsneubildung in der Arterienintima von den mechanischen Bedingungen des Blutumlaufes. Virchows Arch., Bd. 93, 1883.

13. Westphalen: Histologische Untersuchungen über den Bau einiger Arterien. Diss., Dorpat 1886. 
368 Dr. M. S. M a s loff: Die Entwicklung der grossen Gefässe etc.

\section{Erklärung der Abbildungen auf Tafel XVII.}

Fig. 1. Aorta abdominalis eines Embryo von $3,1 \mathrm{~cm}$ Länge. $\mathrm{Zeiss,} \mathrm{Obj.} \mathrm{D,} \mathrm{Komp.-Okul.} \mathrm{12.} \mathrm{Fürbung} \mathrm{nach} \mathrm{Hart.} \mathrm{1.} \mathrm{Eine}$ Reihe von Zellen, die dem Endothelium entsprechen. 2. Blutkörperchen, welche Kerne enthalten. 3. Die elastischen Fasern im Embryonalzustand. 4. Die Zellen der Media. 5. Die Adventitia und das umgebente Gewebe.

Fig. 2. A orta ascendens eines $E m b r y o$ yon $;, 1 \mathrm{~cm} L$ änge. Zeiss, Obj. D, Komp-Okul. 4. Färbung nach F rïukel. 1. Endothelium. 2. Tunica media. 3. Adventitia. 4. Elastische Fasern.

Fig. 3. A orta ascendens eines Embryovon $5,8 \mathrm{~cm}$ Länge. Zeiss, Obj. D. Komp-Okul. 4. Fürbung mit Hïmatoxylin-Eosin. 1. Die Endothelialschicht. 2. Blutkörperchen. 3. Tunica media. (Die Adventitia ist nicht dargestellt.)

Fig. 4. Aorta abdominalis eines Embryo von 7,5 cm Liinge. Z e iss, Obj. D, Komp.-Okul. 4, Färbung mit Hämatoxylin - Eosin. 1. Die Schicht der Endothelialzellen. 2. Feinfiserige Subendothelialschicht. 3. Die Membrana elistica interna. 4. Rote Blutkörperchen.

Fig. 5. Aorta ascendens eines Embryovon $17 \mathrm{~cm}$ L $\ddot{i} n g$ e. \%eiss, Obj. D, Komp.-Okul. 4. Fürbung nach Hart. 1. Dis Endothelium. 2 Die Subendothelialschicht. 3. Elastische Fasern. \& Membrima elastica interna. 5 . Tunica media. 6. Adventitia. ‘. Vasa vasorum. 